Monatsschrift für Geburtshülfe und Gynaekologie 1902;16:I-VI

\title{
Contents, Vol. 16, 1902
}

Inhalts -Verzeichnis.

Opig $\cdot$ inalarbeiten.

Abraham, Otto, Zur Behandlung der weiblichen Gonorrhoe

mit Hefe $\quad 1017$

Ahlefelder, C, Klinische und anatomische Beiträge zur

Genitaltuberkulo.se des Weibes. (Hierzu Tafel V-VII.) 296

Ahlfeld, F., Die Zuverlässigkeit der Heisswasser-Alcohol-

Händedesinfection 848

Amann jr., J. A., Zur Technik der traøsperitonealen Exstir-pation des carcinomatösen Uterus mit Beckenaus-räumang, mit besonderer Berücksichtigung der Ureter-deckung und der Drainage der Beckenhòhle . . . 290

- Zur Frage der weiblichen Genitaltuberkulose .... 586

Bßrndt, Fritz, Ein Fall von grosser Blasenscheidenfistel

mit fast völligem Defekt der Harnröhre. Wiederher-

stellang der Funktion 875

Bondi, Josef, Ueber den Ban der Nabelgefässe. (Hierzu

Tafel IY)

265

Brü $\mathrm{n}$ in $\mathrm{g}, \mathrm{H}$ er $\mathrm{m}$ ann, Tuberculose der weiblichen Geschlechts-

organe ím Kindesalter 144

B uc ura, Constantin J., Ueber die Bedentung des Schüttel-

frostes im Wochenbett mit besonderer Berücksichtigung

der Pyämie 705

Basse, Otto, Ueber die Biklung der Hämatocelen ... 1

Cursehmann, Fritz, Bietet der quere Fundalschnitt bei

der Sectio caesarea (G. Fritsch) gegenüber dem Längs-

schnitt durch die Corpuswand Vorteile? . 172, 772, 882 Czerwenka, K., Beitrag zur

pathologischen Anatomie der

Arulvitis (Pruritus vulvae) 1013

Dützmann, Diagnose und Behancllung der Exsudate . . 71 Fehling, H, Die Hysterectomie in der

Behandluog der

puerperalen Infektion 506

Fellner, Otfried, Ueber den Blutdruck in Seliwangerschaft

und Geburt $\quad 852$

Hertzka, Josef, Ueber das Eindringen von Badewasser in

die Scheide von Schwangeren und Gebärenden und

über die Zweckinässigkeit des Bades bei denselben 821 Hofmeier, M., Die Indikationen zur

künstlichen Unter-

brechung der Schwangerschaft wegen Nephritis . 468

Hoeven, P. C. ï. van der, Junge menschliche Eier . . 6 
Kermauner, Fritz, Scîi $\Lambda$ vangerschaft und Geburtskom])likationen bei Atresie und Stenose der Scheide . . 868

- $\quad$ Ein Fall von Placentarretention mit Untersuchung der retinirten Eitheile $\quad \ldots \ldots . . \mathrm{v} 975$

- $\quad$ Notiz zu meinem Aufsatz: Schwangerschaft und Geburts- : v

komplikationen. bei Atresie und Stenose der Scheide 1029

IV -

Khautz jr., A. v., Eine seltene Form von Ovarial-Dermvid 78

Koch, Jos., Typhusbacillen in der Tube 19S

Kraus, Emil, Nachweis von Gonokokken in den tiefen

Schichten der Tubenwand. (Híerzu Tafel I) ... 192 Krebs, Julius, Ueber Gebärmutterzerreissung während der

Geburt 204

Krönig, Zur Frage der Verwertbarkeit der Lungenschwimm-

probe bei Keimgehalt der Uterusliöhle. (HierzuTafel III) 818 Leopold, G., Die Hysterectomie bei der Puerperal-Infektion 514 Loennberg, Ingolf, Ein Fall von ungewöhnlich kleinem

Foetus compressus 25

Martin, A., Ueber Genitaltuberkulose 555

Mueller, A., Zur Kasuistik der Positio verticalis (Mittel-

scheitellage Kehrers) $84<\mathrm{S}$

Pine us, Ludwig, Das Verhältnis der Atmokausis und

Zestokausis zur Curettage und ihre Heilfaktoren . . 745 Reidhaar, Beitrag zur Behandlung der puerperalen Sepsis 765

Runge, M., Ein Wort zur Hebammenreform 326

Schauta, Friedrich, Die Einleit $\pi$ ng der Geburt wegen

innerer Erkrankungen 470

Schultz, Walther, Ueber Ovarienverptlanzung .... $981>$ Semon, M. , Ueber Komplikation der

Geburt mit üvarial-

tumoren. Ovariotomia abdominalis inter partum mit

nachfolgender Entbindung per vias naturales. (Hierzu

Tafel II) Solowij, A., Zur Kenntnis der pathologischen Anatomie der

Placenta 7

Szili, Alexander, Ein Fall von Adenofibrom des Liga-

mentum rotundum (Hierzu Tafel VIII) 979

Thiemisch, Martin, Ueber die Storch'sche Reaktion der

Frauenmilch , 32

Veit, J., Ueber Tuberkulose der weil > lichen Sexualorgane

und des Peritoneum 525

Wertheim, E., Die chirurgische Behandlung des Uterus-

carcinoms 576

Wiener, Gustav, Ein Adenomyom mit papillären Auf-

lagerungen 131

Zimmermann, Victor, Die intrauterine Ballonbehandlung

in der Geburtshülfe 37

$<\mathrm{i}$

tn 
Bepiehte.

Bacon, C. S., Berícht über die amerikanische geburtshilflichgynaekologische Litteratur mit Angabe ihrer Hauptquellen .407

Ehrenfest, Hugo, Quartalsbericht über die geburtshülfliche und gynaekologische Litteratur der Vereinigten Staaten (April, Mai, Juni 1902) 1037

Graefe, M.. Neuere ArBeiten über Nervenleiden und

Störuftgen des weiblichen Geschlechtsorganes . . . 1030 $-\mathrm{V}-$

Hantke, R. , Die medicinischen Indikationen zur Unterbrechung der Schwangerschaft $\quad 370$

Havtz, A., Ueber die Tuberkulose der weiblichen Genitaloi gane330̃

Hübl, Hugo, Ueber künstlicke Sterilisirung des Weibes 82, 237

Kevmauner, Fritz, Ueber die Anatomie uiul Aetiologie

der Blasenniole 225

Lecène, Aus der franzòsischen Litteratur im le-tzten

Quartal 190192

Mathes, P., Was leistet die Methode der Gefrierpunktsbestimmuog? 787

Monchy, M. M. de, Die geburtshülflieheund gynaekologische

Litteratur in Holland 1902. I. Quartal" 249

Neugebauer, Franz, Achter Bericht über die polnische geburtshülflich-gynaekologische Litteratur (das Jahr 1901 umfassend) 924,1045

Palm, Hermann, Referat über das Mutterkorn .... 912

Törngen, Adolf, Bericht über die fiimländiseke gynae-

kologisch-geburtshülfliche Litteratur. I. Semester 1902791

Der IV. Kongress der Internationalen Gesellschaft far Geburtshülfe und Gynaekologie 417

Epilog des IV. internationalen Kongresses für Geburtshülfe und Gynaekologie in Rom 630

Bericht über den IV. Internationalen Kongress für Geburts

hülfe und Gynaekologie in Rom 633

Die gynaekologische Sektion der 74. Naturforscher-Ver-

sammlung in Karlsbad 1902666

74. Versammlung Deutscher Naturforscher und Aerzte in

Karlsbad. Abteilung für Geburtshülfe und Gynae

kologie667

Nachtrag zum Bericht über den römischen Kongress . 697, 951

NekroJogee.

ïheodor von Kézsmárszky $\uparrow 96$

Edoardo Porro † . . ‘ 419

Akiupg $\Gamma$ isehes; Thepapeutisehes; Pepsonalien und Tag-esnachpichten.

Akiurgisches 254 
Therapeutisches

252

Personalien und Tagesnachrichten $\quad \ldots \quad .95,254,418$

Vereins- und Litteraturbeilage.

Opiglnalsitzung ·sbepichte aus grebuptshülf lieh-gynaekolog · iscfren Gesellsehaften und Vepeinen.

Pommersche gynaekologische Gesellschaft . . 99, 811, 1070

Gynaekologische Gesellschaft in München . '.'.’ 107, 437, 796

$\mathrm{VI}-$

Hussische Gesellschaí't für Geburtshülfe and Gynaekologie

422, 822, 938, 1059 Gesellschaft für Geburtshülfe mid Gynaekologie zu Köln . 444

Referate aus geburtshülf lieh-gynaekologischen Gesellschaften.

Referate aus geburtshülflich-gynaekologischen Ge

sellschaften $113,256,453,828,956,1070$

Gebuptshülllich-gynaekologische Mitteilungen aus andepen Gesellschaften und Vereinen.

Geburtsh ülflic h-gynaekologische Mitteilungen aus

anderen Gesellschaften undYereinen 118,259,830,963,

1078

Buehanzeigen.

Ballantyne, J. W., Manual of Antenatal Pathology and

Hygiene of the foetus 121

Bergholm, Hjalmar, Ueber Mikroorganismen des Yaginal-

secretes Schwangerer 1081

Bericht über die vom Komitee für Krebsforschung am

15. Oktober 1900 erhobene Sammelforschung . . . 458

Bumm, E., Grundriss zum Studium cler Geburtshülfe

Oh.robak, H, Berichte aus der zweiten geburtshülflich-

gynaekolögischen Klinik in VVien 834

(Jouvelaire, A., Hématome du cordon onibilical .... 1080 Kahlden, C. v., Ueber die kleincystische

Degeneration der

Ovarien und ihre Beziehungen zu dem sog. Hydrops

folliculi $\quad \therefore$ '...967

Küstner, O., Kurzes Lehrbuch der Gynaekologie . . 1082

Lennander, Acute (eitrige) Peritonitis96í3 3

Neugebauer, Franz, Interessante Beobachtungen aus dem ;

Gebiete des Scheinzwittertumes 459

Olshausen und Yeit, Lehrbuch der Geburtshülfe . . . 455 Stoeckel, W., Weitere Erfahrungen über

Ureterfistehi und

Ureterverletzungen 1080

Varnier, H, Observation et autopsie d'une femme qui

prétendait s'être fait avorter en s'introduisant une sonde,

dans l'utérus 1079

Veit, s, Olshausen u. V.

Vogel, G., Leitfaden der Geburtshülfe für praktische Aerzte

und Stuclierende $\quad 968$

Walthard, M., Die bakteriotoxische Endometritis . . 122

Litteratup-Vepzeiehnis. 
L·itteratur-Vèrzeichnis . .- . 125,262,460,837,970,1083 